\title{
Comparative outcomes in cardiogenic shock patients managed with Impella microaxial pump or extracorporeal life support
}

\author{
Yoan Lamarche, MD, ${ }^{\mathrm{a}}$ Anson Cheung, MD, ${ }^{\mathrm{a}}$ Andrew Ignaszewski, MD, ${ }^{\mathrm{a}}$ Jennifer Higgins, $\mathrm{MD},{ }^{\mathrm{a}}$ \\ Annemarie Kaan, MCN RN, ${ }^{\mathrm{a}}$ Donald E. G. Griesdale, MD, MPH, ${ }^{\mathrm{b}}$ and Robert Moss, MD ${ }^{\mathrm{a}}$
}

\begin{abstract}
Objective: Cardiogenic shock is associated with poor clinical outcomes. Extracorporeal life support is used in most centers for short-term circulatory support. Alternatively, the Impella LP 5.0 and right direct (RD) microaxial ventricular assist device (Abiomed, Danvers, Mass) can provide isolated left and right ventricular support, respectively.
\end{abstract}

\begin{abstract}
Methods: A retrospective, single center review was performed on all patients receiving circulatory assistance with either extracorporeal life support or Impella ventricular assist device. All Impella LP 5.0 were inserted via the femoral artery, while the RD system required sternotomy.
\end{abstract}

\begin{abstract}
Results: Twenty-nine patients received ventricular assist device support (Impella LP 5.0; $\mathrm{n}=24$; and Impella $\mathrm{RD} ; \mathrm{n}=5$ ), whereas 32 patients were placed on extracorporeal life support. The baseline characteristics of patients with cardiogenic shock, assisted by Impella or extracorporeal life support, were similar, but the etiology of cardiogenic shock was distributed differently in the 2 groups $(P=.008)$. Forty-one percent of the Impella patients and $47 \%$ of the extracorporeal life support patients were weaned from support. The 30-day mortality (44\% in the extracorporeal life support vs $38 \%$ in the Impella group) and proportion of patients discharged home ( $41 \%$ in the extracorporeal life support vs $59 \%$ in the Impella group) were not statistically different between the 2 groups. Arterial thrombosis was less frequent in the Impella group $(3.4 \%$ vs $18.8 \% ; P=.04)$. Blood product transfusions were less frequent in the Impella group $(P<.001)$.
\end{abstract}

Conclusions: Both extracorporeal life support and axial flow pumps provided adequate support in patients with various etiologies of cardiogenic shock. Axial-flow pump may be an optimal type of support for patients with univentricular failure, whereas extracorporeal life support could be reserved for patients with biventricular failure or combined respiratory and circulatory failure. (J Thorac Cardiovasc Surg 2011;142:60-5)

Cardiogenic shock in the postcardiotomy setting after myocardial infarction or as a manifestation of acute decompensated heart failure is associated with high mortality. Low cardiac output syndrome leads to systemic hypoperfusion, which, if not reversed, is followed by multiple organ dysfunction syndrome and death. Treatment options available when patients present with low cardiac output states include revascularization, correction of surgical pathology, and mechanical support of the failing heart. Mechanical circulatory support systems include intra-aortic balloon counterpulsa-

From St. Paul's Hospital, ${ }^{\text {a }}$ Providence Health Care, Vancouver, British Columbia,

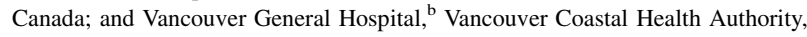
Affiliated Teaching Hospitals of The University of British Columbia, Vancouver, British Columbia, Canada.

Dr Griesdale is supported in part by the members of the Vancouver Hospital Department of Anesthesia, and through a Clinician Scientist Award from the Vancouver Coastal Health Research Institute.

Disclosures: Authors have nothing to disclose with regard to commercial support.

Received for publication March 23, 2010; revisions received July 6, 2010; accepted for publication July 31, 2010; available ahead of print Sept 29, 2010.

Address for reprints: Yoan Lamarche, MD, 1081 Burrard St, St. Paul's Hospital, Vancouver, BC, Canada V6Z 1Y6 (E-mail: yoanlamarche@gmail.com).

$0022-5223 / \$ 36.00$

Copyright (C) 2011 by The American Association for Thoracic Surgery doi: $10.1016 /$ j.jtcvs.2010.07.075 tion (IABP), ventricular assist devices (VADs), and extracorporeal life support (ECLS), also called veno-arterial extracorporeal membrane oxygenation (ECMO). Isolated right ventricular failure is rare after cardiac surgery or myocardial infarction, and it requires management with fluid optimization, inotropes, and pulmonary vasodilators. Despite aggressive management, including right ventricular support devices, its mortality ranges from 50 to $60 \%{ }^{1}$

In an effort to decrease the invasiveness of the devices used for support, smaller temporary VADs have become available in the form of endovascular axial flow pumps for the right and left ventricle. The Impella recover system (Abiomed, Danvers, Mass) includes pumps that can be inserted peripherally, such as the left peripheral $(2.5 \mathrm{~L}$ or 5.0 L [LP2.5 and LP5.0]), or centrally for the left is the left direct (LD) or for the right is the right direct (RD) ventricular support. ${ }^{2-4}$ The use of these devices has been reported by several centers, although mostly in the form of small case series or case reports. ${ }^{3-8}$ In the postcardiotomy setting, Impella support has been compared, in a retrospective analysis, to intra-aortic balloon counterpulsation. ${ }^{9}$ The use of axialflow pumps has also been reported in several trials as support for high-risk percutaneous coronary interventions. ${ }^{10-14}$ However, most of these trials compare the Impella LP2.5 


$$
\begin{aligned}
& \text { Abbreviations and Acronyms } \\
& \begin{aligned}
& \text { CPR }=\text { cardiopulmonary resuscitation } \\
& \text { ECLS }=\text { extracorporeal life support } \\
& \text { ECMO }=\text { extracorporeal membrane oxygenation } \\
& \text { eCPR }=\text { mechanically assisted cardiopulmonary } \text { resuscitation } \\
& \text { IABP }=\text { intra-aortic balloon counterpulsation } \\
& \text { IQR }=\text { interquartile range } \\
& \text { LV }=\text { left ventricular } \\
& \text { PRBC }=\text { packed red blood cells } \\
& \text { RD }=\text { right direct } \\
& \text { RV }=\text { right ventricle } \\
& \text { VAD }=\text { ventricular assist device }
\end{aligned}
\end{aligned}
$$

device, with a maximal output of $2.5 \mathrm{~L} / \mathrm{min}$, and IABP. We believe that there are no reports comparing Impella $5.0 \mathrm{~L}$ devices with ECLS, in the setting of cardiogenic shock as a bridge to recovery, to a longer-term VAD, or as a bridge to decision strategy.

The objective of this study is to compare survival, weaning, and bridging outcomes, as well as bleeding and thromboembolic complications of consecutive patients supported by the newly introduced microaxial VAD systems with ECLS in patients with severe acute cardiogenic shock in a single center.

\section{PATIENTS AND METHODS}

A retrospective cohort was constructed of all patients requiring mechanical circulatory support in the setting of acute, refractory, cardiogenic shock at St. Paul's Hospital between July 2000 and September 2009. St. Paul's Hospital is a 500-bed quaternary care hospital affiliated with the University of British Columbia and offers both interventional and cardiac surgical care. Patients presenting with acute ST segment elevation myocardial infarctions were directed according to protocolized care to the catheterization laboratory for percutaneous intervention with surgical backup. Patients with postcardiotomy shock were evaluated for residual surgical defects, and correction was performed by either a surgical or percutaneous approach. All patients having potential for recovery were considered for mechanical support if their systemic perfusion did not improve with IABP and inotropes. Potential for recovery was described as absence of irreversible multiorgan dysfunction and was evaluated by consensus between the surgeons and cardiologists from the heart failure team. Etiology and severity of shock was described by cause and by the extent of the pharmacologic and mechanical support required, including IABP and cardiopulmonary resuscitation (CPR). Patients requiring cardiopulmonary resuscitation continuously, intermittently, or on very high doses of vasopressors and inotropes (norepinephrine $>20 \mu \mathrm{g} / \mathrm{min}$ or unstable despite three or more vasopressors and/or inotropes) were considered as undergoing mechanically assisted cardiopulmonary resuscitation (eCPR). Patients with cardiogenic shock with echocardiographic signs of isolated right ventricular failure (distension, left-sided displacement of interventricular septum, and severe tricuspid regurgitation) were considered for support with ECLS (2000-2009) or right-sided axial flow pump (Impella RD, 20072009). Demographics, diagnosis, type of support, and flow data were obtained from a registry containing perioperative information of all patients requiring mechanical support. Complications and outcomes were obtained by chart revision and institutional database for discharge status.

\section{Support Systems}

The only support system available in patients failing despite IABP therapy from 2000 to 2007 was ECLS. From October 2007 to September 2009, both ECLS and Impella systems were available.

The ECLS system was composed of Carmeda coated cannulae and circuit (Biomedicus; Medtronic, Minneapolis, Minn). Cannula size varied and ranged from 17 to 23 French and 20 to 29 French for arterial and venous access, respectively. The oxygenator and heat exchanger were also heparin-coated (Quadrox D oxygenator Maquet, Hirrlingen, Germany). A Biomedicus centrifugal pump head was used initially and was replaced with the Rotaflow pump (Maquet) in more recent cases. Patients requiring emergency support in the cardiac catheterization laboratory received percutaneous veno-arterial cannulations. Patients in the postcardiotomy setting had either central or peripheral veno-arterial cannulation. Heparin was used during cannulation and weaning only. No left ventricular vent was inserted in patients supported with ECLS.

The Impella Recover system is composed of an axial-flow pump driven by an electric motor. The Impella LD and LP 5.0 system consists of a 21French inflow tip positioned into the left ventricle and the outflow in the aorta. The LP system was introduced in the femoral artery after a vascular cut down, either through a direct canulation of the artery through a pursestring or through a vascular graft. Once the pump was positioned under fluoroscopy into the left ventricle, the 9-French driveline was connected to the console. Patients supported by the RD system had direct right atrial and main pulmonary artery canulation through median sternotomy, with purse string sutures ensuring hemostasis around the inflow and outflow cannulae. Intravenous heparin was given at the time of pump insertion to obtain an ACT $>300$ seconds. During support, a partial prothrombin time (PTT) of 45 to 60 seconds was maintained with continuous heparin infusion.

\section{Selection and Management of Devices}

Between 2007 and 2009, both the Impella systems and ECLS were available. Patients with biventricular failure and oxygenation problems were supported by ECLS. Patients with unilateral ventricular failure were supported with the Impella systems.

Transesophageal echocardiography provided assistance during both device insertion and explantation, as well as during patient's circulatory support as needed. Systematic transthoracic echocardiograms were performed every 48 hours during support to assess myocardial recovery, the presence of intracardiac thrombus, and device placement. Thromboembolic complications were considered when thrombotic material was identified in the arterial system, or when a limb or an organ became clinically ischemic secondary to thrombus formation, embolism, or suspected physical obstruction by a cannula.

Patients were assessed for recovery of organ failure and neurologic complications. If no signs of myocardial recovery were present, patients were considered for an implantable long-term VAD if they were transplant candidates.

\section{Statistical Analysis}

Data is presented as median \pm interquartile range (IQR) or mean \pm standard deviation. Categorical data was compared with $\chi^{2}$ analysis or Fisher exact test where appropriate. Mann-Whitney $U$ Test was used for continuous variables. All statistical evaluations were performed using Stata version 11 (StataCorp, College Station, Tex). A statistically significant difference was defined as a two-sided $P$ value less than .05 .

This study was approved by the clinical research ethics board at St. Paul's Hospital and the University of British Columbia.

\section{RESULTS}

During the 9-year study period, 7952 patients had open heart surgery at St. Paul's Hospital. There were 142 heart 
transplantations and 52 implantable, long-term VADs performed. During the same period, 32 patients required ECLS. Twenty-four patients $(0.3 \%$ of cases $)$ necessitated ECLS after cardiac surgery. Eight patients were supported with ECLS in the setting of mechanical assistance to resuscitation. Twenty-nine patients were supported with axial flow pumps (Impella LP 5.0; $\mathrm{n}=24$ and Impella RD; $\mathrm{n}=5$ ). The mean age, gender, proportion of patients with diabetes mellitus, preoperative IABP, as well as the initial left ventricular ejection fraction was similar between the two groups (Table 1). There was twice as many patients requiring support as a mechanical assistance to resuscitation or as an adjunct to CPR (eCPR) in the Impella group $(P=$ not significant). Seventy-five percent of ECLS cases were in the postcardiotomy setting, whereas only $21 \%$ of the Impella systems were inserted for postcardiotomy ventricular failure. All patients in the ECLS group and 28 $(97 \%)$ of the Impella group were mechanically ventilated when the decision to support them was established. Patients had an ischemic etiology in $41 \%$ and $38 \%$ of the ECLS and Impella cases, respectively (Table 1). However, the other etiologies of shock were different in the 2 groups $(P=.008)$, with the proportion of postcardiotomy shock being higher in the ECLS group.

Forty-seven and $41 \%$ of the ECLS and Impella patients, respectively, were successfully weaned from mechanical support (Table 2 and Figure 1). One patient from the Impella group required the addition of veno-venous ECMO for hypoxemic respiratory failure. Five patients had isolated rightsided axial flow pump (Impella RD) inserted for severe right heart failure. Four of those patients $(80 \%)$ were weaned and were subsequently discharged from the hospital. Arterial thrombotic events were more frequent in patients receiving ECLS ( $18.8 \%$ vs $3.4 \% ; P=.04)$. The arterial thromboembolic events included 1 left ventricular thrombus and 5 episodes of leg ischemia, related to the cannulation site in the ECLS group; in the axial flow pump group, 1 patient was diagnosed with heparin-induced thrombocytopenia with internal jugular vein thrombosis, mitral valve thrombosis, and femoral artery thrombosis requiring surgery.

The 30-day mortality (44\% in ECLS vs $38 \%$ in the Impella group) and proportion of patients discharged home ( $41 \%$ in ECLS vs 59\% in the Impella group) was not statistically different between the 2 groups. There were no cases of mechanical failure requiring replacement or altered medical management in the Impella group, whereas replacement of circuit component was needed in $9 \%$ of ECLS cases.

Blood products were used less frequently in the Impella group than in the ECLS group $(P<.001)$. The patients supported after cardiotomy required more packed red blood cells (PRBC) transfusions than patients supported in all other categories (18 U [IQR, 10-34) vs 4 U [IQR, 2-9]; $P<.0001)$. Use of activated recombinant factor VII was
TABLE 1. Patient characteristics: extracorporeal life support, intraaortic balloon pump, ejection fraction, standard deviation, mechanically supported resuscitation, and idiopathic dilated cardiomyopathy

\begin{tabular}{lccc}
\hline & $\begin{array}{c}\text { ECLS } \\
(\mathbf{n}=\mathbf{3 2})\end{array}$ & $\begin{array}{c}\text { Impella } \\
(\mathbf{n = 2 9 )}\end{array}$ & $\boldsymbol{P}$ \\
\hline Age (mean \pm SD) & $50.4 \pm 14.2$ & $53.7 \pm 13.1$ & .35 \\
Male gender (\%) & $62.5 \%$ & $82.8 \%$ & .08 \\
Diabetes (\%) & $20.0 \%$ & $27.6 \%$ & .49 \\
Mechanically ventilated (n) (\%) & $32(100)$ & $28(97)$ & .29 \\
Preoperative IABP (\%) & $40.6 \%$ & $51.7 \%$ & .39 \\
eCPR (n) (\%) & $8(25)$ & $14(48)$ & .06 \\
Mean EF (\%) & $20(13-25)$ & $20(11-20)$ & .54 \\
Cause of shock & & & $\mathbf{. 0 0 8}$ \\
IDCM (\%) & 3.1 & 24.1 & \\
Ischemic (\%) & 40.6 & 37.9 & \\
Myocarditis (\%) & 0 & 10.3 & \\
Postcardiotomy (\%) & 43.8 & 13.8 & \\
Other* (\%) & 12.5 & 13.8 & \\
\hline
\end{tabular}

$E C L S$, Extracorporeal life support; $e C P R$, mechanically supported resuscitation; $E F$, ejection fraction; $I A B P$, intra-aortic balloon pump; IDCM, idiopathic dilated cardiomyopathy; $S D$, standard deviation. *The category "Other" includes in the extracorporeal life support (ECLS) patients: One refractory ventricular arrhythmia, 1 failing left ventricular assist device, 1 decompensated hypertrophic cardiomyopathy, and 1 peripartum cardiomyopathy. In the Impella patients: 2 postcardiac transplants with right ventricle failure, 1 myocarditis, and 1 refractory ventricular arrhythmia patient.

not statistically different between groups $(21.8 \%$ in ECLS group vs $13.8 \%$ in the Impella group; $P=.51$ ) (Table 3 ).

\section{DISCUSSION}

The main findings of this analysis comparing ECLS and axial-flow pump for circulatory support in patients with acute cardiogenic shock are a decreased use of allogenic blood product transfusions and fewer thromboembolic complications in the axial-flow pump group. There was no difference in 30-day mortality. Finally, although not statistically significant, $50 \%$ more patients in the Impella

TABLE 2. Outcomes: ventricular assist device, extracorporeal life support, interquartile range, and standard deviation

\begin{tabular}{lccc}
\hline & $\begin{array}{c}\text { ECLS } \\
(\mathbf{n = 3 2})\end{array}$ & $\begin{array}{c}\text { Impella } \\
(\mathbf{n = 2 9 )}\end{array}$ & $\boldsymbol{P}$ \\
\hline $\begin{array}{l}\text { Duration of support (hr) } \\
\quad(\text { median } \pm \text { IQR) }\end{array}$ & $46.3(27-88)$ & $63.3(41-142)$ & .16 \\
$\begin{array}{l}\text { Average flow (L/min) } \\
\quad(\text { mean } \pm \text { SD) }\end{array}$ & $4.0 \pm 0.1$ & $3.7 \pm 0.1$ & .06 \\
$\begin{array}{l}\text { Arterial thromboembolism } \\
\quad(\mathrm{n})(\%)\end{array}$ & $6(18.8)$ & $1(3.4)$ & .04 \\
Weaned (n) (\%) & & & \\
Bridge to VAD (n) (\%) & $6(18.8)$ & $8(27.6)$ & .41 \\
$\begin{array}{l}\text { Bridge to transplant (n) }(\%) \\
\text { 30-day mortality (n) }(\%)\end{array}$ & $3(9.4)$ & 0 & .09 \\
Discharged home (n) $(\%)$ & $14(43.8)$ & $11(37.9)$ & .64 \\
\hline
\end{tabular}

$E C L S$, Extracorporeal life support; $I Q R$, interquartile range; $S D$, standard deviation; $V A D$, ventricular assist device. 


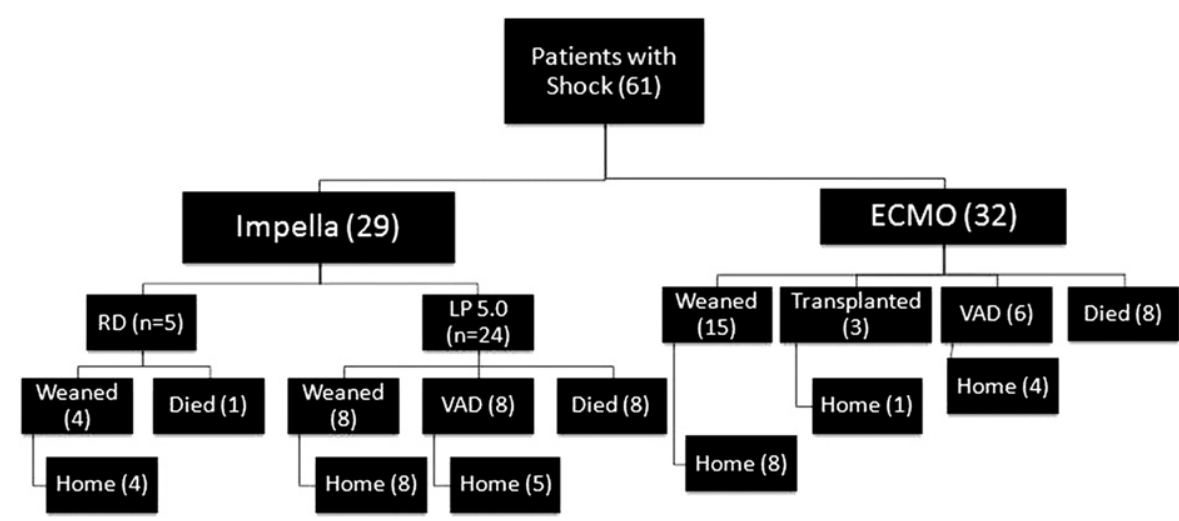

FIGURE 1. Flow diagram. ECLS, Extracorporeal life support; $E C M O$, extracorporeal membrane oxygenation; $L P$, left peripheral; $R D$, right direct; $V A D$, ventricular assist device.

group than the ECLS group were able to be discharged home.

The two groups of patients described in this study had profound cardiogenic shock that was refractory to IABP and inotropes, and all except 1 patient were mechanically ventilated. They were supported by two devices with different physiological effects, and the overall 30-day mortality of $44 \%$ and $38 \%$ was lower than previously reported in this patient population. ${ }^{9,10,15}$ Smedira and colleagues ${ }^{16}$ reported a 30-day mortality of $62 \%$ in cardiogenic shock patients supported with ECLS. Siegenthaler and colleagues ${ }^{9}$ observed $54 \%$ mortality in patients with postcardiotomy low cardiac output syndrome. Jaski and colleagues ${ }^{17}$ reported a survival of $26 \%$ in patients supported by ECLS for shock or cardiac arrest. The cohort presented in this study is composed of patients that had markers of shock that were more profound than patients from other recent series. In their meta-analysis, comparing IABP and percutaneous devices for cardiogenic shock, Cheng and colleagues ${ }^{10}$ described patients that were not all ventilated or on vasopressors, with mean left ventricular ejection fractions between $19 \%$ to $31 \%{ }^{18-20}$ Despite being less supported and having better left ventricular (LV) function, $45 \%$ and $43 \%$ of the patients supported by VADs or IABP did not survive 30 days.

Patients described in the present study were in severe cardiogenic shock, refractory to IABP and vasopressor sup-

TABLE 3. Blood product use: Extracorporeal life support, packed red blood cells, and fresh frozen plasma

\begin{tabular}{lccc}
\hline & $\begin{array}{c}\text { ECLS } \\
(\mathbf{n}=\mathbf{3 2})\end{array}$ & $\begin{array}{c}\text { Impella } \\
(\mathbf{n}=\mathbf{2 9})\end{array}$ & $\boldsymbol{P}$ \\
\hline PRBC (median [IQR]) & $18.0(9-34)$ & $4(2-9)$ & $<.001$ \\
FFP (median [IQR]) & $14(8-28)$ & $2(0-8)$ & $<.001$ \\
Platelets (median [IQR]) & $5(0.5-8.5)$ & $0(0-2)$ & $<.001$ \\
Factor VIIa (\%) & 21.8 & 13.8 & .51 \\
\hline$E C L S$, Extracorporeal life support; $F F P$, fresh frozen plasma; $P R B C$, packed red \\
blood cells.
\end{tabular}

port, and a significant proportion of patients required CPR before initiation of support. Those patients required extensive hemodynamic support using Impella $5.0 \mathrm{~L}$ or complete cardiopulmonary bypass with ECLS as opposed to partial support, such as Tandem Heart or Impella $2.5 \mathrm{~L}$, which is reported in most series.

Support with ECLS has been used for more than 20 years in the postcardiotomy setting ${ }^{21}$ and more recently as a adjunct to CPR. ${ }^{22}$ The physiologic effects of ECLS include unloading of the right ventricle, gas exchange, and systemic perfusion. The left ventricle is only partially unloaded while on ECLS, and LV afterload may be high. Left ventricular venting and IABP have been suggested as being options to optimize LV preload and afterload in case of LV distension with mixed results. ${ }^{22,23}$ In this series, both patients supported by Impella and ECLS had a similar proportion supported by IABP preoperatively, but all IABPs were removed once the mechanical support was instituted. Support with left-sided axial flow pump has the potential advantages of providing systemic perfusion while adequately decompressing the left ventricle and augmenting coronary perfusion. ${ }^{24}$ The flow with both left- and rightsided axial flow pumps is nonpulsatile. Both systems have the potential for peripheral insertion, but the obstruction to blood flow to the lower limb is less with the Impella system (9 French vs 17-23 French).

Patients receiving support with the axial flow pump required fewer transfusions, despite the requirement of systemic heparin. This may be explained by fewer postcardiotomy patients in the Impella group. However, this may also be a consequence of the minimal surgical access through femoral cut down and a reduced activation of inflammatory and coagulation cascades. The reduction of bleeding led the clinical team to be more comfortable with therapeutic anticoagulation. This could explain the significant decrease in peripheral thromboembolism in that group. The short support time on ECLS and the $44 \%$ surgical re-exploration for bleeding or tamponade explains our strategy of avoiding 
anticoagulation in that group. Despite this strategy, we observed only one case of left-sided intracardiac thrombosis as compared with Muehrcke and colleagues ${ }^{25}$ who reported as much as $20 \%$ intracardiac thrombosis when anticoagulation was avoided during ECLS. The potential limitation of the peripheral ischemic episodes by addition of systemic anticoagulation remains speculative. Seyfarth and colleagues ${ }^{19}$ also reported the absence of thromboembolic complications in their Impella group, although they also reported increased bleeding compared to IABP. Significant bleeding was also reported in $52 \%$ of the surgical patients by Granfeldt and colleagues ${ }^{5}$ in their retrospective analysis of 50 patients with Impella support; however, most of their patients required central cannulation with the Impella LD system. We also observed decreased bleeding in patients who were not in a postcardiotomy setting; this finding may be a confounder explaining some of the differences in PRBC transfusions between the ECLS and Impella groups in this cohort of patients. Pages and colleagues ${ }^{26}$ also reported transfusion requirements when comparing ECLS and biventricular VAD support for cardiogenic shock. Interestingly, they observed a mean PRBC transfusion rate of 22 and 7 in the biventricular VAD and ECLS groups, respectively. In that series, none of the ECLS patients were in a postcardiotomy setting or required central canulation, which was also identified as a risk factor for transfusion in the present series. The median duration of support with ECLS or axial flow pumps is widely variable. Granfeldt and colleagues ${ }^{5}$ had a mean support time of 7.3 days and 30-day mortality in the postcardiotomy cohort of $45 \%$. Siegenthaler and colleagues ${ }^{9}$ supported patients for an average of 61 hours and had a mortality of $54 \%$. In the patient supported by Impella reported in the present study, the median support of less than 3 days may imply that many patient had signs of recovery relatively early after restoration of organ perfusion. Siegenthaler and colleagues ${ }^{9}$ also suggested a low risk of mortality (10\%) for patients with residual cardiac function as opposed to poor outcomes ( $88 \%$ mortality) in patients with residual cardiac output of less than $1 \mathrm{~L} / \mathrm{min} .{ }^{9}$ In the present study, $47 \%$ and $41 \%$ of patients were weaned from support in the ECLS and Impella groups, respectively. The weaning was guided by echocardiographic signs of ventricular recovery, which was believed to be a better indicator of cardiac function than thermodilution measured cardiac output in patients with various degrees of right ventricle (RV) dysfunction and tricuspid regurgitation.

The 30-day survival patients supported for isolated RV failure in the Impella group was $75 \%$ in contrast with the results of Granfeldt and colleagues 5 who observed $75 \%$ mortality in 8 patients supported with this system and with mortality of $57 \%$ reported by Moazami and colleagues ${ }^{1}$ in 30 postcardiotomy patients requiring mechanical support. However, the presence of only 5 patients in the isolated RV failure subgroup of the present study, with some being supported in the postcardiotomy setting, after myocardial infarction or after transplant, make the comparison difficult with those other 2 small cohorts.

Following its introduction in 2007, Impella rapidly became the dominant mechanism of hemodynamic support at our institution. This is evident as during the time where these 2 technologies were concurrently available, ECLS was used in 5 patients compared with 29 patients in whom the Impella was inserted. It is likely that both changes in practice and potential benefits of Impella lead to a broadening of the potential indications for hemodynamic support. This is suggested by an increase in mechanical support for cardiomyopathy during this time. In addition, having a postcardiotomy mechanical backup also permits postoperative mechanical support, and facilitates the surgical treatment of higher risk cases. Finally, good initial results with the Impella likely lowered the threshold for univentricular mechanical support.

This retrospective series, to our knowledge, is the largest comparative series of patients requiring full support with mechanical devices including axial flow pumps. In comparison to ECLS, we have observed that the Impella device may reduce patient morbidity with less systemic embolism and transfusion requirement. A nonstatistically significant trend toward improvement in successful hospital discharge was also noted. From a resource utilization perspective, the management of patients with the microaxial VADs did not need 24-hour onsite coverage with perfusionists, which was necessary with ECLS. The ability of the Impella system to relieve ventricular distension is a potential advantage over other modes of support, and it also provides support that is targeted to the failing ventricle and optimizes coronary perfusion.

There are several limitations to this study that need to be addressed. Confounding by indication remains a significant source of bias in the present study as selection for which modality of circulatory support was not random and was based on various patient characteristics. It would be very difficult to adjust for all of these factors, particularly given the small numbers of this study. However, patients in both groups were relatively similar as both had profound cardiogenic shock that was refractory to IABP and inotropes, and all except 1 patient were mechanically ventilated. The increasing numbers of patients surviving the acute decompensation event and becoming transplant or long-term VAD recipients led to an increasing awareness of the heart failure program from the referring physicians. The earlier referral pattern may have led to earlier implantation of support devices later in the series. Finally, as with all observational research, and selection bias, unmeasured and residual confounding remains an alternate explanation for our results.

\section{CONCLUSIONS}

Despite early revascularization, correction of mechanical problems, use of IABP, and inotropes, the mortality of patients with cardiogenic shock remains high. Aggressive circulatory support of high-risk patients with ECLS or 
axial-flow pumps is feasible and was associated with acceptable results. The ease of use, minimal transfusion requirements and low incidence of arterial thrombosis observed with axial flow pumps were identified as advantages for the use of this technology in patients with univentricular failure. The ECLS remains the short-term support of choice in patients with shock from biventricular failure and respiratory insufficiency.

The authors would like to acknowledge and thank Karin Humphries, PhD, Bryan Chow, Jennifer Kealy, Jamil Bashir, MD and James Abel, MD for their contributions and assistance in the preparation of this research and subsequent article.

\section{References}

1. Moazami N, Pasque MK, Moon MR, et al. Mechanical support for isolated right ventricular failure in patients after cardiotomy. J Heart Lung Transplant. 2004; 23:1371-5.

2. Vercaemst L, Vandezande E, Janssens P, Yvan T, Peter D, Meyns B. Impella: a miniaturized cardiac support system in an era of minimal invasive cardiac surgery. J Extra Corpor Technol. 2002;34:92-100.

3. Meyns B, Dens J, Sergeant P, Herijgers P, Daenen W, Flameng W. Initial experiences with the Impella device in patients with cardiogenic shock-Impella support for cardiogenic shock. Thorac Cardiovasc Surg. 2003;51:312-7.

4. Sugiki H, Nakashima K, Vermes E, Loisance D, Kirsch M. Temporary right ventricular support with Impella Recover RD axial flow pump. Asian Cardiovasc Thorac Ann. 2009; 17:395-400.

5. Granfeldt H, Hellgren L, Dellgren G, et al. Experience with the Impella recovery axial-flow system for acute heart failure at three cardiothoracic centers in Sweden. Scand Cardiovasc J. 2009;43:233-9.

6. Jung C, Ferrari M, Rodiger C, Fritzenwanger M, Figulla HR. Combined Impella and intra-aortic balloon pump support to improve macro- and microcirculation: a clinical case. Clin Res Cardiol. 2008;97:849-50.

7. Martin J, Benk C, Yerebakan C, Derjung G, Sarai K, Beyersdorf F. The new "Impella" intracardiac microaxial pump for treatment of right heart failure after orthotopic heart transplantation. Transplant Proc. 2001;33:3549-50.

8. Samoukovic G, Al-Atassi T, Rosu C, Giannetti N, Cecere R. Successful treatment of heart failure due to acute transplant rejection with the Impella LP 5.0. Ann Thorac Surg. 2009;88:271-3.

9. Siegenthaler MP, Brehm K, Strecker T, et al. The Impella Recover microaxial left ventricular assist device reduces mortality for postcardiotomy failure: a threecenter experience. J Thorac Cardiovasc Surg. 2004;127:812-22.

10. Cheng JM, den Uil CA, Hoeks SE, et al. Percutaneous left ventricular assist devices vs. intra-aortic balloon pump counterpulsation for treatment of cardiogenic shock: a meta-analysis of controlled trials. Eur Heart J. 2009;30:2102-8.
11. Cohen R, Foucher R, Sfaxi A, Hakim M, Domniez T, Elhadad S. [High-risk left main coronary stenting supported by percutaneous Impella Recover LP 2.5 assist device.] Ann Cardiol Angeiol (Paris). 2010;59:48-51.

12. Dixon SR, Henriques JP, Mauri L, et al. A prospective feasibility trial investigating the use of the Impella 2.5 system in patients undergoing high-risk percutaneous coronary intervention (The PROTECT I Trial): initial U.S. experience. JACC Cardiovasc Interv. 2009;2:91-6.

13. Ramondo A, Napodano M, Tarantini G, et al. High-risk percutaneous coronary intervention using the intracardiac microaxial pump 'Impella recover'. J Cardiovasc Med (Hagerstown). 2006;7:149-52.

14. Thomopoulou S, Manginas A, Cokkinos DV. Initial experience with the Impella Recover LP 2.5 micro-axial pump in patients undergoing high-risk coronary angioplasty. Hellenic J Cardiol. 2008;49:382-7.

15. Hsu PS, Chen JL, Hong GJ, et al. Extracorporeal membrane oxygenation for refractory cardiogenic shock after cardiac surgery: predictors of early mortality and outcome from 51 adult patients. Eur J Cardiothorac Surg. 2010;37:328-33.

16. Smedira NG, Moazami N, Golding CM, et al. Clinical experience with 202 adults receiving extracorporeal membrane oxygenation for cardiac failure: survival at five years. J Thorac Cardiovasc Surg. 2001;122:92-102.

17. Jaski BE, Ortiz B, Alla KR, et al. A 20-year experience with urgent percutaneous cardiopulmonary bypass for salvage of potential survivors of refractory cardiovascular collapse. J Thorac Cardiovasc Surg. 2010;139:753-7.

18. Burkhoff D, Cohen H, Brunckhorst C, O'Neill WW. A randomized multicenter clinical study to evaluate the safety and efficacy of the TandemHeart percutaneous ventricular assist device versus conventional therapy with intraaortic balloon pumping for treatment of cardiogenic shock. Am Heart J. 2006;152:469-8.

19. Seyfarth M, Sibbing D, Bauer I, et al. A randomized clinical trial to evaluate the safety and efficacy of a percutaneous left ventricular assist device versus intraaortic balloon pumping for treatment of cardiogenic shock caused by myocardial infarction. J Am Coll Cardiol. 2008;52:1584-8.

20. Thiele H, Sick P, Boudriot E, et al. Randomized comparison of intra-aortic balloon support with a percutaneous left ventricular assist device in patients with revascularized acute myocardial infarction complicated by cardiogenic shock. Eur Heart J. 2005;26:1276-83.

21. Conrad SA, Rycus PT, Dalton H. Extracorporeal Life Support Registry Report 2004. ASAIO J. 2005;51:4-10.

22. Chen B, Chang YM. CPR with assisted extracorporeal life support. Lancet. 2008 372:1879-80.

23. Magovern GJ Jr, Magovern JA, Benckart DH, et al. Extracorporeal membrane oxygenation: preliminary results in patients with postcardiotomy cardiogenic shock. Ann Thorac Surg. 1994;57:1462-8.

24. Remmelink M, Sjauw KD, Henriques JP, et al. Effects of left ventricular unloading by Impella recover LP2.5 on coronary hemodynamics. Catheter Cardiovasc Interv. 2007;70:532-7.

25. Muehrcke DD, McCarthy PM, Stewart RW, et al. Complications of extracorporeal life support systems using heparin-bound surfaces. The risk of intracardiac clot formation. J Thorac Cardiovasc Surg. 1995;110:843-51.

26. Pages ON, Aubert S, Combes A, et al. Paracorporeal pulsatile biventricular assist device versus extracorporal membrane oxygenation-extracorporal life support in adult fulminant myocarditis. J Thorac Cardiovasc Surg. 2009;137:194-7. 